Supporting Information

\title{
REACTIVE TANDEM ION MOBILITY SPECTROMETRY WITH ELECTRIC FIELD FRAGMENTATION OF ALCOHOLS AT AMBIENT PRESSURE
}

\author{
Hossein Shokri, Maika Vuki, ${ }^{1}$ Ben Gardner, ${ }^{2}$ Hsein-Chi Niu, ${ }^{2}$ Umesh Chiluwal, Bhupendra K. Gurung, \\ David B. Emery, and Gary A. Eiceman \\ Department of Chemistry and Biochemistry \\ New Mexico State University \\ Las Cruces, NM 88003 \\ ${ }^{1}$ Chemistry Department \\ University of Guam \\ Mangilao, Guam 96913 \\ ${ }^{2}$ Collins Aerospace, Pomona, CA 91767
}

Corresponding author: Gary A. Eiceman, email address: geiceman@nmsu.edu 
Supporting Information

Table S1. Supporting information for Figure 5 (data supplement Table 1).

\begin{tabular}{|c|c|c|c|c|c|c|c|c|c|}
\hline \multirow{2}{*}{ Compound } & $t_{d}$ & Ko & $t_{d}$ & $\mathrm{~K}_{\mathrm{o}}$ & $t_{d}$ & Ko & $\Delta t_{d}$ & $\Delta \mathrm{K}_{\mathrm{o}}$ & \multirow{2}{*}{$\begin{array}{c}\text { Ion } \\
\text { Mass }(\mathrm{g} / \mathrm{mol}) \\
\mathrm{MH}^{+}\left(\mathrm{H}_{2} \mathrm{O}\right)_{\mathrm{n}}\end{array}$} \\
\hline & \multicolumn{2}{|c|}{$\mathrm{MH}^{+}\left(\mathrm{H}_{2} \mathrm{O}\right)_{\mathrm{n}}$} & \multicolumn{2}{|c|}{$\mathrm{M}_{2} \mathrm{H}^{+}$} & \multicolumn{2}{|c|}{ Fragment ion } & \multicolumn{2}{|c|}{$\mathrm{MH}^{+}\left(\mathrm{H}_{2} \mathrm{O}\right)_{n}$ - Fragment ion } & \\
\hline isopropyl acetate & 4.85 & 1.80 & 6.35 & 1.38 & 4.14 & 2.11 & 0.71 & 0.31 & 139.13 \\
\hline butyl acetate & 5.11 & 1.72 & 6.90 & 1.28 & 4.15 & 2.12 & 0.96 & 0.40 & 153.16 \\
\hline isopentyl acetate & 5.46 & 1.61 & 7.54 & 1.17 & 4.10 & 2.15 & 1.36 & 0.54 & 167.19 \\
\hline pentyl acetate & 5.52 & 1.60 & 7.60 & 1.16 & 4.14 & 2.13 & 1.38 & 0.53 & 167.19 \\
\hline hexanal & 5.18 & 1.68 & 6.62 & 1.31 & 4.78 & 1.82 & 0.40 & 0.14 & 137.16 \\
\hline heptanal & 5.50 & 1.58 & 7.22 & 1.21 & 4.88 & 1.79 & 0.62 & 0.21 & 151.18 \\
\hline octanal & 5.80 & 1.50 & 7.78 & 1.12 & 5.25 & 1.66 & 0.55 & 0.16 & 165.21 \\
\hline nonanal & 6.14 & 1.42 & 8.30 & 1.05 & 5.39 & 1.62 & 0.75 & 0.20 & 179.24 \\
\hline octane & 4.91 & 1.77 & - & - & 3.89 & 2.24 & 1.02 & 0.47 & 151.23 \\
\hline nonane & 5.16 & 1.69 & - & - & 4.02 & 2.17 & 1.14 & 0.48 & 165.20 \\
\hline decane & 5.43 & 1.60 & - & - & 4.20 & 2.07 & 1.23 & 0.47 & 179.29 \\
\hline
\end{tabular}

Drift Time, $t_{d}$, in ms.

Reduced mobility coefficient, $\mathrm{K}_{\mathrm{o}}$, in $\mathrm{cm}^{2} / \mathrm{Vs}$ 
Supporting Information

Table S2. Influence of Electric Field Strength on Percent Fragmentation of Protonated Monomer

\begin{tabular}{|c|c|c|c|c|c|c|c|c|}
\hline & \multicolumn{6}{|c|}{ Percent Fragmentation at $67^{\circ} \mathrm{C}$ and Field Strength $(\mathrm{E} / \mathrm{N})$} & \multirow{2}{*}{\multicolumn{2}{|c|}{$\begin{array}{c}\text { Terms for Linear } \\
\text { Regression of Percent } \\
\text { Fragmentation vs E/N }\end{array}$}} \\
\hline & $69 \mathrm{Td}$ & $77 \mathrm{Td}$ & $90 \mathrm{Td}$ & $99 \mathrm{Td}$ & $112 \mathrm{Td}$ & $120 \mathrm{Td}$ & & \\
\hline & \multicolumn{6}{|c|}{ Normal alcohols } & Slope & Intercept \\
\hline 1-propanol & 0.0 & 0.6 & 2.0 & 1.9 & 5.1 & 16.5 & 0.181 & -13.28 \\
\hline 1-butanol & 0.0 & 2.5 & 12.2 & 15.6 & 25.8 & 42.5 & 0.772 & -56.52 \\
\hline 1-pentanol & 8.4 & 11.4 & 34.4 & 46.5 & 58.8 & 65.6 & 1.199 & -75.76 \\
\hline 1-hexanol & 7.6 & 7.4 & 26.0 & 35.0 & 51.4 & 54.6 & 1.029 & -66.95 \\
\hline 1-heptanol & 10.8 & 16.9 & 29.5 & 37.0 & 49.2 & 54.4 & 0.876 & -49.81 \\
\hline 1-octanol & 9.7 & 17.3 & 20.4 & 39.4 & 44.2 & 50.5 & 0.818 & -47.03 \\
\hline \multirow[t]{2}{*}{ 1-nonanol } & 0.2 & 1.3 & 8.2 & 30.0 & 27.9 & 33.7 & 0.663 & -45.17 \\
\hline & \multicolumn{6}{|c|}{ Other alcohols } & & \\
\hline cyclohexanol & 23.6 & 38.8 & 51.3 & 70.4 & 80.4 & 93.8 & 1.332 & -66.18 \\
\hline 2-butanol & 7.6 & 13.8 & 26.0 & 35.0 & 42.1 & 51.1 & 0.818 & -47.03 \\
\hline isobutanol & 3.1 & 14.5 & 25.6 & 28.3 & 45.2 & 56.1 & 0.973 & -63.15 \\
\hline
\end{tabular}


Supporting Information
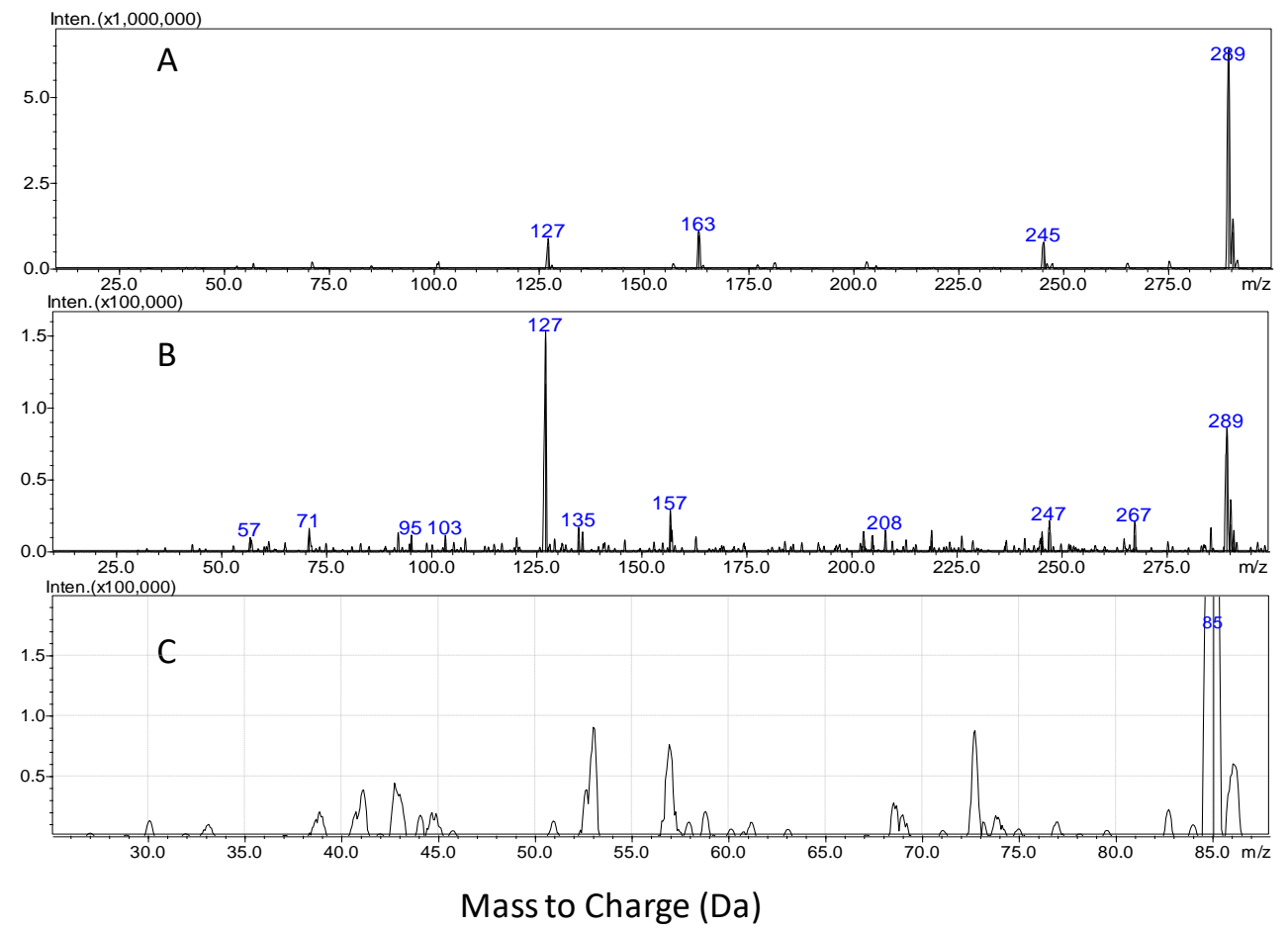

Figure S1. Mass spectra for 1-nonanol with a drift tube-mass spectrometer at $60^{\circ} \mathrm{C}$. Spectra are shown without (A) and with (B) electric field fragmentation at $168 \mathrm{Td}$. In Frame $\mathrm{C}$, an expanded mass axis is shown for ions of low abundance ions from 25 to $90 \mathrm{Da}$. 


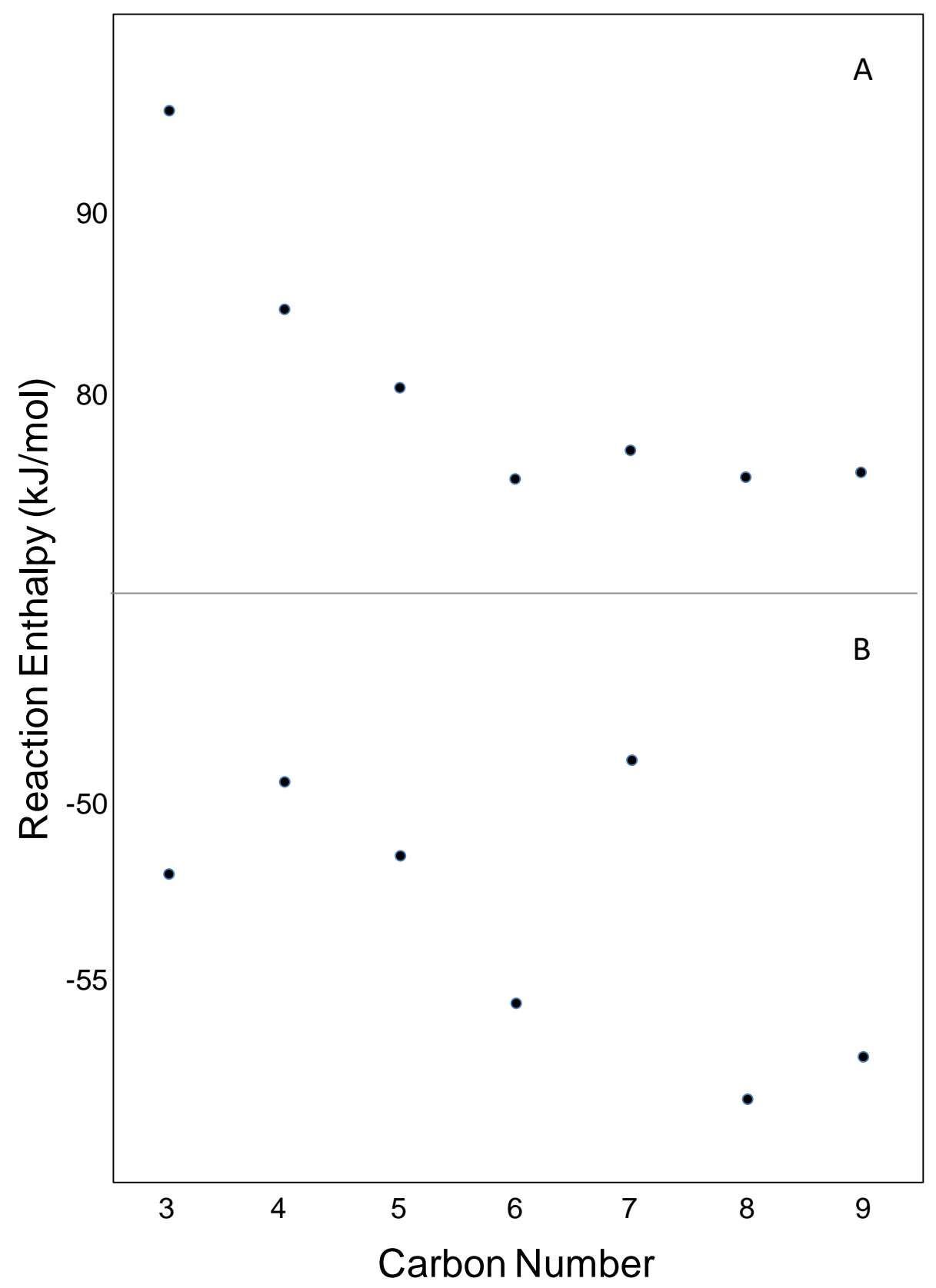

Figure S2. Enthalpy of fragmentation of normal alcohols in: A- a first step of water elimination from protonated monomer to a primary carbocation and B- a second step of charge migration to a secondary carbocation. 